\title{
Simulação como estratégia de interferência na autoconfiança interprofissional no âmbito da atenção primária
}

\author{
Simulation as interference strategy in interprofessional self-confidence in primary health care \\ Simulación como estrategia de inteferencia en la autoconfianza interprofesional en el ámbito de la \\ atención primaria
}

\section{Maria Beatriz Silva Siqueira', Breno de Sousa Santana", Bárbara Soares Rodrigues ${ }^{\prime \prime}$, Marcia Cristina da Silva Magro ${ }^{\prime V}$}

\begin{abstract}
RESUMO
Objetivo: verificar se a estratégia de simulação empregada para capacitação em suporte básico de vida e situações de obstrução de vias aéreas interfere na autoconfiança interprofissional no âmbito da atenção primária à saúde. Método: estudo quaseexperimental desenvolvido com 68 profissionais da atenção primária do Distrito Federal, entre junho e dezembro de 2018. Utilizaram-se questionários para caracterização do conhecimento e avaliação da autoconfiança. Realizou-se aula expositiva e simulação realística sobre parada cardiopulmonar e obstrução de vias aéreas como intervenção, sendo significativos os resultados com diferença estatisticamente significativa antes e após $(p<0,05)$. Resultados: amostra majoritariamente feminina $(69,1 \%)$, com idade entre 21 e 70 anos, com predomínio de agentes comunitários de saúde $(32,4 \%)$. Houve melhora na autoconfiança para o manejo correto da parada cardiopulmonar e da desobstrução de vias aéreas $(p<0,001)$ após a intervenção. Conclusão: a simulação favorece aprendizagem experiencial, desenvolvendo autoconfiança interprofissional para lidar com emergências na atenção primária à saúde.
\end{abstract}

Descritores: Treinamento por simulação; educação interprofissional; autoconfiança; atenção primária à saúde.

\section{ABSTRACT}

Objective: to verify if the simulation strategy employed for basic life support training and situations of airway obstruction interferes with interprofessional self-confidence in primary health care. Method: quasi-experimental study conducted with 68 primary care professionals in the Federal District, Brazil, between June and December 2018. Questionnaire to characterize knowledge and self-confidence were used. A lecture and realistic simulation on cardiopulmonary arrest and airway obstruction were performed as intervention, being statistically significant different results before and after intervention $(p<0,05)$. Results: mostly female sample (69.1\%), aged between 21 and 70 years. Community health agents predominated (32.4\%). Self-confidence improved for the correct management of cardiopulmonary arrest and airway clearance $(p<0.001)$ after intervention. Conclusion: Simulation supports experiential learning, developing interprofessional self-confidence in dealing with emergencies in primary health care.

Descriptors: Simulation training; interprofessional education; self-confidence; primary health care.

\section{RESUMEN}

Objetivo: verificar si la estrategia de simulación empleada para el entrenamiento de soporte vital básico y las situaciones de obstrucción de las vías respiratorias interfieren con la autoconfianza interprofesional en la atención primaria de salud. Método: estudio cuasi experimental realizado con 68 profesionales de atención primaria en el Distrito Federal, Brasil, entre junio y diciembre de 2018. Se utilizó un cuestionario para caracterizar el conocimiento y la autoconfianza. Una intervención y una simulación realista sobre el paro cardiopulmonar y la obstrucción de las vías aéreas se realizaron como intervención, siendo resultados estadísticamente significativos diferentes antes y después de la intervención $(p<0,05)$. Resultados: muestra mayoritariamente femenina $(69,1 \%)$, con edades comprendidas entre 21 y 70 años. Predominaron los agentes comunitarios de salud $(32,4 \%)$. La autoconfianza mejoró para el manejo correcto del paro cardiopulmonar y el despeje de la vía aérea $(p<0.001)$ después de la intervención. Conclusión: La simulación apoya el aprendizaje experimental, desarrollando la autoconfianza interprofesional para enfrentar emergencias en la atención primaria de salud.

Descriptores: Entrenamiento simulado; educación interprofesional; autoconfianza; atención primaria de salud.

\section{INTRODUÇÃo}

A educação interprofissional (EIP) é uma importante abordagem pedagógica, que prepara os profissionais para fornecer atendimento ao paciente por uma equipe colaborativa. Comprovadamente, equipes qualificadas de profissionais são capazes de proporcionar um atendimento mais seguro, reduzir os custos e os erros associados a assistência à saúde ${ }^{1}$.

'Graduanda em Enfermagem. Faculdade de Ceilândia, Universidade de Brasília. E-mail: maria_beatrizsilva@ hotmail.com

"Enfermeiro especialista. Mestrando da Faculdade de Ciências da Saúde, Universidade de Brasília. E-mail: bresousas@outlook.com

I'Enfermeira especialista. Mestranda da Faculdade de Ciências da Saúde, Universidade de Brasília. E-mail: mio.barby@hotmail.com

IVEnfermeira. Doutora em enfermagem. Professora adjunta da Faculdade de Ceilândia, Universidade de Brasília. E-mail: marciamagro@unb.br

vAgradecimento à Liga de Simulação em Saúde (LISSA) da Faculdade de Ceilândia da Universidade de Brasília (FCE/UnB) pela parceria e apoio na realização do estudo. 
Definida como uma forma de aprendizagem em que estudantes ou membros de duas ou mais profissões aprendem uns com os outros, para melhorar a colaboração e a qualidade dos cuidados, visa aprimorar atitudes, conhecimentos e habilidades para a prática colaborativa, que por sua vez podem melhorar a prática clínica ${ }^{2,3}$.

A integração entre a EIP e o aprendizado simulado tem sido identificada como um fator-chave na transformação do ensino de enfermagem ${ }^{4}$. Nessa direção, a educação baseada em simulação visa criar uma experiência orientada para ajudar na aquisição das habilidades necessárias e garantir a segurança e o bem-estar dos pacientes ${ }^{5}$.

Sendo assim, potencializar competências e habilidades em tempo real tem se tornado um desafio, possível de ser alcançado através da estratégia de simulação, assim como aperfeiçoar técnico e cientificamente o profissional para alcance de conhecimentos desejáveis à segurança do cuidado ${ }^{6}$, não subestimando a autoconfiança, habilidade não técnica essencial para a tomada de decisões acertadas e julgamento crítico em contexto clínico ${ }^{7}$. Sabidamente, as unidades básicas de saúde (UBS) compreendem o ponto de apoio à população acometida por doenças crônicas e, sendo assim, uma equipe multiprofissional competente pode ser o diferencial para minimizar ou solucionar agravos e complicações em todos os níveis de saúde ${ }^{8}$.

Entretanto, não raro, profissionais da atenção primária à saúde (APS) se deparam com pacientes em situações graves, que podem evoluir rapidamente na ausência de intervenções corretas e ágeis. No âmbito da saúde, a qualificação profissional ocupa local de destaque, especialmente quando nos referimos às situações críticas com risco de morte ${ }^{9}$.

Nesse cenário, a parada cardiorrespiratória (PCR) e a obstrução de vias aéreas por corpo estranho (OVACE) ganham destaque e estão entre as situações que exigem da equipe de saúde agilidade e decisões assertivas para estabilização do quadro clínico em qualquer ambiente, sobretudo na APS. Tal situação reforça que o pensamento crítico e rápido não deve ser subestimado no processo de salvar vidas, de modo que a capacitação de uma equipe representa o alvo para a redução de erros e dos índices de mortalidade ${ }^{10}$.

Nessa perspectiva deve ser sublinhado que a necessidade de uma equipe qualificada na APS dotada de profissionais com competências e habilidades suficientes influencia na efetivação da segurança do paciente ${ }^{11}$. Frente a isso, a simulação, ênfase do presente estudo, emerge e se destaca como chave mediadora para alcance de uma aprendizagem experiencial em cenário seguro e supervisionado ${ }^{12}$. Contudo, expor os profissionais da APS a situações emergenciais simuladas, reproduzidas de forma realística por meio de capacitações simuladas revelou-se como uma proposta oportuna ao desenvolvimento da autoconfiança e do raciocínio clínico e crítico, na resolução de problemas.

Nesse contexto, o objetivo do presente estudo foi verificar se há ganho de autoconfiança em situações emergenciais a partir de capacitações mediadas pela a estratégia de simulação no âmbito da atenção primária à saúde.

\section{METODOLOGIA}

Trata-se de um estudo quase-experimental não equivalente desenvolvido com profissionais de saúde atuantes nas unidades básicas de saúde (UBS) da região oeste do Distrito Federal, durante o período de junho a dezembro de 2018. A amostra foi não probabilística de conveniência, constituída de 68 profissionais (enfermeiros, técnicos de enfermagem, agentes comunitários de saúde (ACS), dentistas, psicólogos e médicos). Foram excluídos aqueles em período de férias ou em licença trabalhista e que não preencheram completamente os instrumentos de coleta de dados.

Adotou-se um questionário estruturado com itens de identificação pessoal, conhecimento sobre suporte básico de vida em cardiologia (SBV) e obstrução de vias aéreas por corpo estranho (OVACE) e ainda, uma escala de autoconfiança em emergência do tipo likert de 5 pontos, sendo 1 equivalente a nada confiante e 5 extremamente confiante. Tal escala foi desenvolvida em 2006 e possui validação de conteúdo para a língua portuguesa, com consistência interna demonstrada pelo Alfa de Cronbach global de 0,918 ${ }^{7,13}$. O seu desenvolvimento se associou à necessidade de avaliar a variável autoconfiança de formandos, aqui adaptada para profissionais, em quatro dimensões (1) reconhecimento de sinais e sintomas, (2) avaliação precisa do paciente, (3) intervenção apropriada à demanda e (4) avaliação da eficácia das intervenções, nas esferas respiratória, cardíaca e neurológica.

A intervenção foi uma estratégia combinada teórico-prática, composta de aula expositivo-dialogada e estações de simulação híbrida, combinação de ator vivo e simulador de paciente, para efetivação da capacitação, com objetivo de desenvolver habilidades não técnicas, aqui representada pela autoconfiança no atendimento de situações críticas, para tal adotou-se as diretrizes internacionais de SBV e OVACE ${ }^{14}$ para a equipe multiprofissional fundamentada pela estratégia de educação interprofissional.

A operacionalização da coleta de dados foi orientada por fases. Sendo assim, na Fase 1, como estratégia disparadora foi realizada visita e sensibilização dos gestores das UBS, a fim de obter liberação da equipe multiprofissional em dias estabelecidos em cronograma visando alcançar os profissionais de todas as UBS da região oeste, do Distrito Federal. 
Na Fase 2, aplicação do questionário e da escala de autoconfiança em emergência aos profissionais em ambiente reservado da UBS pelos estudantes da liga acadêmica, sob supervisão docente, com duração de 60 minutos.

Na Fase 3, ministrou-se durante 40 minutos aula expositivo-dialogada com auxílio de multimídia. A intervenção foi desenvolvida por estudantes de uma liga acadêmica especializada em simulação realística, treinados e supervisionada por docentes, com expertise na área de simulação e emergências, de uma instituição de ensino superior pública.

Na Fase 4, foi realizada alocação aleatória e distribuição dos profissionais em grupos constituídos por quatro participantes em três estações práticas: (1) suporte básico de vida adulto; (2) suporte básico de vida pediátrico e (3) obstrução de vias aéreas por corpo estranho. Todas as estações foram conduzidas pelos estudantes ligantes sob supervisão docente em diferentes salas das UBS, de modo a assegurar a privacidade para atuação e discussão da situação clínica proposta. Nessas estações, havia demonstração de práticas simuladas necessárias para aquisição de autoconfiança no atendimento de SBV e OVACE, a partir do emprego de simuladores de pacientes e atores vivos. Ao término, realizou-se o debriefing, momento de reflexão sobre a autoconfiança acerca das práticas vivenciadas em cada estação prática, com duração de 30 minutos.

Na Fase 5, pós-intervenção, reaplicou-se a escala de autoconfiança para avaliação do perfil de autoconfiança póscapacitação, com duração de 60 minutos.

Os dados foram tabulados em planilhas do Microsoft Excel ${ }^{\circledast} 2016$ e analisados com auxílio do Statistical Package for the Social Sciences (SPSS) versão 23. A distribuição de normalidade foi avaliada pelo teste Kolmogorov Smirnov. Realizou-se análise descritiva com cálculo de medidas resumo (frequências absoluta, relativa e mediana) e medidas de dispersão (percentil 25 e 75). Na análise estatística, dada distribuição assimétrica, empregaram-se o teste de MannWhitney para a comparação entre grupos e Wilcoxon Signed Ranks para a comparação intragrupos. Considerados significativos os resultados com valor de $p<0,05$.

A todos os profissionais foi garantido anonimato, bem como resguardado o direito de declinar da pesquisa em qualquer fase. Todos expressaram aceite de participação através da assinatura do Termo de Consentimento Livre e Esclarecido (TCLE). O estudo foi aprovado por Comitê de Ética em Pesquisa sob o parecer número 1.207.279 e CAEE 47489415.8.0000.5553.

\section{RESULTADOS E DISCUSSÃO}

Os resultados desse estudo mostraram a efetividade do emprego da estratégia de simulação realística no desenvolvimento de autoconfiança dos profissionais da APS, sobre SBV e OVACE. As características dos participantes do estudo são apresentadas na Tabela 1.

Dos 68 profissionais participantes, a maioria enquadrou-se no cargo de agente comunitário de saúde (ACS) $(32,4 \%)$, técnico de enfermagem (20,6\%), enfermeiro $(14,7 \%)$ e médico $(13,2 \%)$. Embora a idade dos profissionais tenha variado entre 21 e 70 anos, houve predomínio da faixa etária de 31 a 40 anos. A maioria era do sexo feminino (69,1\%), com tempo de formação de 5 a 10 anos (35,3\%). Do total, 30,9\% dos profissionais declararam ter cursado especialização (pós-graduação lato sensu) e o tempo de exercício profissional na APS foi predominantemente entre 5 a 10 anos.

Os dados relativos à avaliação da autoconfiança interprofissional e na comparação com os ACS são apresentados na Tabela 2.

Identificou-se que a autoconfiança dos profissionais para atender situações envolvendo SBV e OVACE melhorou significativamente $(p<0,001)$, progredindo de $2,3(1,9-3,0)$ na etapa pré-intervenção para $3,0(2,7-3,4)$ na etapa pósintervenção.

No contexto da APS, este estudo identificou que os agentes comunitários de saúde (ACS) apresentaram menor autoconfiança para prestar atendimento em emergências quando comparados aos demais profissionais de saúde, e essa diferença foi significativa nos momentos pré $(p=0,001)$ e pós-intervenção $(p=0,005)$. Embora pós-intervenção tenha se verificado melhora expressiva da autoconfiança entre os profissionais ACS $(p=0,002)$ e Não-ACS $(p<0,001)$.

A autoconfiança dos profissionais em situações relacionadas ao cuidado direcionado a situações que necessitavam de SBV e OVACE mostrou melhora progressiva. A capacitação permite que profissionais quando expostos a uma situação simulada, próxima ao real, adquira a chance de aprender fazer, a partir de instruções e esclarecimentos reduzindo o risco de danos ao paciente ${ }^{10}$

Um estudo realizado no hospital infanto-juvenil do Estado do Paraná, concluiu que a simulação favoreceu o desenvolvimento de autoconfiança por mediar a aproximação de gestores junto as equipes de trabalho, melhorar a comunicação, analisar situações de conflitos, superar barreiras, desenvolver atitudes e habilidades específicas, além de prover um ambiente de maior segurança e confiança para a realização dos procedimentos, permitindo a identificação e a reconstrução de conceitos e condutas $^{15}$. 
TABELA 1: Características sociodemográficas e de formação dos profissionais de saúde das Unidades Básicas de Saúde. Brasília, DF, Brasil, 2018 (N=68).

\begin{tabular}{|c|c|c|}
\hline Variáveis & $\mathbf{n}$ & $f(\%)$ \\
\hline Sexo feminino & 47 & 69,1 \\
\hline \multicolumn{3}{|l|}{ Faixa Etária } \\
\hline 21 H 30 anos & 7 & 10,3 \\
\hline $31-40$ anos & 31 & 45,6 \\
\hline $41-50$ anos & 20 & 29,3 \\
\hline $51-60$ anos & 5 & 7,4 \\
\hline $61-70$ anos & 3 & 4,4 \\
\hline Não respondeu & 2 & 2,9 \\
\hline \multicolumn{3}{|l|}{ Formação } \\
\hline Agente Comunitário de Saúde & 22 & 32,4 \\
\hline Técnico de Enfermagem & 14 & 20,6 \\
\hline Médico & 9 & 13,2 \\
\hline Enfermeiro Assistencial & 8 & 11,8 \\
\hline Dentista & 6 & 8,8 \\
\hline Técnico de Saúde Bucal & 3 & 4,4 \\
\hline Enfermeiro Gerente & 2 & 2,9 \\
\hline Técnico Administrativo & 2 & 2,9 \\
\hline Não respondeu & 2 & 2,9 \\
\hline \multicolumn{3}{|l|}{ Tempo de formação (anos) } \\
\hline $5 H 10$ anos & 24 & 35,3 \\
\hline 11 H 15 anos & 19 & 27,9 \\
\hline $1 H 5$ anos & 5 & 7,4 \\
\hline até 1 ano & 2 & 2,9 \\
\hline Não respondeu & 5 & 7,4 \\
\hline \multicolumn{3}{|l|}{ Pós-graduação lato sensu } \\
\hline Especialização & 21 & 30,9 \\
\hline Outros & 1 & 1,5 \\
\hline Não respondeu & 46 & 67,6 \\
\hline \multicolumn{3}{|c|}{ Tempo de exercício profissional na atenção primária à saúde } \\
\hline $5 H 10$ anos & 29 & 42,6 \\
\hline $11 H 15$ anos & 12 & 17,6 \\
\hline $1-5$ anos & 10 & 14,7 \\
\hline Até 1 ano & 7 & 10,3 \\
\hline Mais de 15 anos & 5 & 7,4 \\
\hline Não respondeu & 5 & 7,4 \\
\hline
\end{tabular}

TABELA 2: Diferença da evolução da autoconfiança no atendimento interprofissional a emergências na atenção primária do período pré para o período pós-intervenção. Brasília, DF, Brasil, 2018 (N=68).

\begin{tabular}{lllr}
\hline & $\begin{array}{l}\text { Pré-intervenção } \\
\text { Mediana (percentis 25-75) }\end{array}$ & $\begin{array}{l}\text { Pós-intervenção } \\
\text { Mediana (percentis 25-75) }\end{array}$ & p \\
\hline $\begin{array}{l}\text { Avaliação geral } \\
\text { Avaliação ACS x Demais profissionais }\end{array}$ & $2,3(1,9-3,0)$ & $3,0(2,7-3,4)$ & $<0,001^{*}$ \\
Não-ACS $(n=46)$ & $2,5(2,0-3,0)$ & $3,2(2,8-4,0)$ & $<0,001^{*}$ \\
ACS $(n=22)$ & $1,9(1,3-2,2)$ & $2,9(2,5-3,0)$ & $0,002^{*}$ \\
& $p=0,001^{* *}$ & $p=0,005^{* *}$ & \\
\hline
\end{tabular}

Notas: ACS - Agente Comunitário de Saúde.

*Teste Wilcoxon Signed Ranks; **Teste Mann-Whitney.

Achado similar foi identificado no presente estudo, onde a estratégia de simulação permitiu o desenvolvimento da autoconfiança na prática clínica. Condição essencial quando se pensa na equipe multiprofissional, que atua na APS, local com ampla demanda e vigente necessidade de capacitação para atualização de condutas a partir de diretrizes baseadas em evidências, assim como a consolidação do trabalho em equipe visando assegurar um cuidado mais confiável e seguro ${ }^{16}$. 
Além disso, a importância da simulação como capacitação interprofissional pode ser aplicada tanto para enfermeiros quanto para técnicos, auxiliares e até mesmo para a equipe de outros setores, como das unidades administrativas, gestores, executivos, administradores hospitalares, reguladores e legisladores. Para estes grupos, acredita-se que a simulação possa reproduzir as complexidades do trabalho clínico, e então ser adotada para o exercício das práticas organizacionais das instituições clínicas ${ }^{15,17}$, como desenvolvido no presente estudo.

Para os profissionais, a prática de simulação é uma excelente oportunidade para compartilhar conhecimento, trocar experiências e aprimorar habilidades. Estudos que utilizaram a estratégia de simulação realística como veículo de capacitação profissional afirmam que foi possível identificar falhas no conhecimento, dificuldades na execução dos procedimentos, necessidades de aprendizagem, capacitação e revisão de técnicas, estratégia que tem permitido a reconstrução de saberes por meio dos questionamentos e reflexões ${ }^{18,19}$.

As simulações quando realísticas, por mimetizarem uma atuação real podem contribuir tanto para o aumento da autoconfiança quanto para a redução do nível de ansiedade e medo ${ }^{20,21}$. No presente estudo identificou-se o ganho significativo de autoconfiança acerca do conhecimento das diretrizes de SBV e OVACE. O cenário simulado oferece um ambiente seguro e controlado onde os profissionais de saúde podem reunir a teoria e a prática clínica sem temer prejuízos aos pacientes 22,23 .

Estudo realizado na Califórnia com uma equipe multiprofissional mostrou melhora significativa no tempo de respostas dos profissionais nos primeiros cinco minutos de assistência a emergências, após a capacitação simulada. Destaca-se que entre os cenários eleitos, na fibrilação ventricular, a equipe apresentou principalmente redução no tempo de início das compressões e agilidade na solicitação de ajuda ${ }^{24}$. Tais achados, assim como os do presente estudo, evidenciam o ganho de segurança na atuação profissional, no que se refere a realização de procedimentos de forma mais efetiva, rápida e segura.

A capacitação de uma equipe multiprofissional mediada pela simulação para melhorar o atendimento a pacientes violentos, por exemplo, pode proporcionar ganhos na ampliação de habilidades e ainda contribuir aos enfrentamentos difíceis da equipe com os pacientes, a partir dos ganhos relacionados ao conhecimento, confiança, preparação, e segurança pessoal ${ }^{25}$. Em nosso estudo, mesmo entre os profissionais sem formação a nível superior, como os agentes comunitários de saúde, houve ganho expressivo da autoconfiança após capacitação para atendimento de emergências.

Ao profissional de saúde, independentemente de sua atribuição exige-se conhecimentos atualizados e constante prática para desenvolvimento de sua autoconfiança durante o processo assistencial. A simulação em grande escala recria os elementos de uma situação para treinamento de equipes multiprofissionais, sendo assim reconhecida como um método efetivo ${ }^{26}$.

O desenvolvimento de autoconfiança é fundamental para a tomada de decisões assertivas e para resolução de processos de julgamento em contexto clínico. Vários estudos mostram que a autoconfiança em emergências aumenta quando estão presentes fatores como a prática repetida e a formação pautada na estratégia de simulação ${ }^{10,27,28}$. Condições oferecidas a equipe multiprofissional da APS para desenvolvimento não somente de competências cognitivas, mas também da autoconfiança possibilita o avanço na prática do cuidado por meio de um processo colaborativo ${ }^{29}$.

Este estudo apresentou como limitações dificuldades técnicas e estruturais nas UBS para implementação da estratégia de simulação, além da ausência de alguns profissionais nos dias previstos para o desenvolvimento da proposta. Apesar disso, foi constatável que a capacitação possibilitou aos profissionais das UBS contato com equipamentos, a exemplo dos simuladores de paciente e conhecimentos atualizados, além de promover reflexão entre gestores e equipe multidisciplinar sobre os avanços e atualizações do processo de cuidado em situações críticas.

\section{CONCLUSÃO}

O método de simulação realística adotado para capacitação dos profissionais direcionado à assistência a situações de PCR e OVACE melhorou a autoconfiança para enfrentamento de situações críticas e de risco.

O desenvolvimento de autoconfiança direcionado às práticas de saúde possibilita o atendimento de qualidade com menor chance de erros. O profissional de saúde, para atuar na prática clínica, precisa estar cientificamente atualizado e seguro em suas ações, para ter agilidade e habilidade no atendimento.

A prática com repetição e dentro de um ambiente próximo ao real, como o simulado, permite ao profissional exercer ações, possibilitando a reflexão como estratégia para agregar conhecimento. A capacitação mediada pela simulação representa uma oportunidade de a equipe multiprofissional compartilhar experiências e aprender de forma segura, tanto habilidades técnicas assim como as não técnicas, aqui representada pela autoconfiança, alvo do presente estudo. 


\section{REFERÊNCIAS}

1. Reeves S. Why we need interprofessional education to improve the delivery of safe and effective care. Interface - Comun Saúde, Educ [Internet]. 2016 [cited 2019 Nov 18]; 20(56):185-97. DOI: https://dx.doi.org/10.1590/1807-57622014.0092

2. WHO. A WHO report: Framework for action on interprofessional education and collaborative practice [Internet]. Vol. 39, Journal of Allied Health. 2010 [cited 2019 Nov 18]. 39:196-7 p. Available from: https://www.who.int/hrh/resources/framework_action/en/

3. Green BN, Johnson CD. Interprofessional collaboration in research, education, and clinical practice: working together for a better future. J. Chiropr. Educ. [Internet] 2015 [cited 2019 Nov 18]; 29(1):1-10. Available from: http://journalchiroed.com/doi/10.7899/JCE-14-36

4. Decker SI, Anderson M, Boese T, Epps C, McCarthy J, Motola I, et al. Standards of best practice: simulation standard VIII: Simulation-Enhanced Interprofessional Education (Sim-IPE). Clin. Simul. Nurs [Internet]. 2015 [cited 2019 Nov 18]; 11(6):293-7. DOI: http://dx.doi.org/10.1016/j.ecns.2015.03.010

5. Lateef F. Simulation-based learning: Just like the real thing. J Emerg Trauma Shock [Internet]. 2010 [cited 2019 Nov 18]; 3(4):348. Available from: http://www.onlinejets.org/text.asp?2010/3/4/348/70743

6. Bordignon M, Monteiro MI. Use of simulation in trianing on violence in nursing practice. Acta Paul. Enferm. [Internet] 2019 [cited 2019 Nov 18]; 32(3):341-9. DOI: https://dx.doi.org/10.1590/1982-0194201900047

7. Martins JCA, Baptista RCN, Coutinho VRD, Mazzo A, Rodrigues MA, Mendes IAC. Self-confidence for emergency intervention: adaptation and cultural validation of the Self-confidence Scale in nursing students. Rev. Latinoam. Enfermagem [Internet]. 2014 [cited 2019 Nov 18]; 22(4):554-61. DOI: https://dx.doi.org/10.1590/0104-1169.3128.2451

8. Schenker M, Costa DH Da. Advances and challenges of health care of the elderly population with chronic diseases in Primary Health Care. Cien. Saude Colet. [Internet]. 2019 [cited 2019 Nov 18] 2; 24(4):1369-80. DOI: https://dx.doi.org/10.1590/141381232018244.01222019

9. Oliveira MA de C, Pereira IC. Primary Health Care essentials attributes and the Family Health Strategy. Rev. Bras. Enferm. [Internet]. 2013 [cited 2019 Nov 18]; 66(spe):158-64. DOI: https://dx.doi.org/10.1590/s0034-71672013000700020

10. Mesquita HCT, Santana BS, Magro MCS. Effect of realistic simulation combined to theory on self-confidence and satisfaction of nursing professionals. Esc Anna Nery Rev. Enferm. [Internet]. 2019 [cited 2019 Nov 18] 24; 23(1):1-6. DOI: https://dx.doi.org/10.1590/2177-9465-ean-2018-0270

11. Farias DC de, Celino SDM, Peixoto JBS, Barbosa ML, Costa GMC. Receptivity and solvability of emergencies in the Family Health Strategy. Rev. Bras. Educ. Med. [Internet]. 2015 [cited 2019 Nov 18]; 39(1):79-87. DOI: https://dx.doi.org/10.1590/198152712015v39n1e00472014

12. Fonseca LMM, Aredes NDA, Fernandes AM, Batalha LMC, Apóstolo JMA, Martins JCA, et al. Computer and laboratory simulation in the teaching of neonatal nursing: innovation and impact on learning. Rev Latinoam. Enfermagem [Internet]. 2016 [cited 2019 Nov 18]; 24:e2808. DOI: https://dx.doi.org/10.1590/1518-8345.1005.2808

13. Hicks FD, Coke L, Li S. Report of findings from the effect of high-fidelity simulation on nursing students' knowledge and performance: a pilot study [Internet]. Chicago, Ill.: National Council of State Boards of Nursing; 2009 [cited 2019 Nov 18]. Available from: https://mafiadoc.com/the-effect-of-high-fidelity-simulation-on-nursing-students-knowledge598f841c1723ddce69c5df24.html

14. American Heart Association. Destaques das atualizações específicas das diretrizes de 2017 da American Heart Association para Suporte Básico de Vida em Pediatria e para Adultos e qualidade da ressuscitação cardiopulmonar. American Heart Association [Internet]. 2017 [cited 2019 Nov 18]; 2. Available from: https://eccguidelines.heart.org/wp-content/uploads/2017/12/2017Focused-Updates_Highlights_PTBR.pdf

15. Abreu AG, Freitas JS, Berte M, Ogradowski KRP, Nestor A. The use of realistic simulation as teaching-learning methodology for nursing staff in a child-adolescent hospital: experience report. Ciência \& Saúde [Internet]. 2014 [cited 2019 Nov 18]; 7(3):162. DOI: https://dx.doi.org/10.15448/1983-652X.2014.3.17874

16. Forster AC, Ferreira JBB, Vicentine FB. Atenção à Saúde da Comunidade no Âmbito da Atenção Primária à Saúde na FMRP-USP [Internet]. 1ạ ed. Ribeirão Preto (SP): Funpec Editora; 2017 [cited 2019 Nov 18]. Available from: https://edisciplinas.usp.br/pluginfile.php/4952445/mod_resource/content/1/Atencao_a_Saude_da_Comunidade.pdf

17. Sanino GE de C. O uso da simulação em enfermagem no Curso Técnico de Enfermagem. J. Heal Inform. [Internet] 2012 [cited 2019 Nov 18]; 4:148-51. Available from: http://www.jhi-sbis.saude.ws/ojs-jhi/index.php/jhi-sbis/article/view/247/136

18. Woods C, West C, Mills J, Park T, Southern J, Usher K. Undergraduate student nurses' self-reported preparedness for practice. Collegian [Internet]. 2015 [cited 2019 Nov 18]; 22(4):359-68. DOI: https://dx.doi.org/10.1016/j.colegn.2014.05.003

19. Costa RRO, Medeiros SM, Martins JCA, Menezes RMP, Araújo MS. The use of simulation in the context of health and nursing education: an academic. Espaço para a Saúde - Rev Saúde Pública do Paraná [Internet]. 2015 [cited 2019 Nov 18]; 16(1):59. DOI: http://dx.doi.org/10.22421/1517-7130.2015v16n1p59

20. Failla KR, Macauley K. Interprofessional simulation: a concept analysis. Clin. Simul. Nurs. [Internet] 2014 [cited 2019 Nov 18]; 10(11):574-80. DOI: http://dx.doi.org/10.1016/j.ecns.2014.07.006

21. Smithburger PL, Kane-Gill SL, Kloet MA, Lohr B, Seybert AL. Advancing interprofessional education through the use of high fidelity human patient simulators. Pharm. Pract. (Granada) [Internet]. 2013 [cited 2019 Nov 18]; 11(2):61-5. DOI: https://dx.doi.org/10.4321/s1886-36552013000200001

22. Generoso JR, Latoures RE, Acar Y, Miller DS, Ciano M, Sandrei R, et al. Simulation training in early emergency response (STEER). J. Contin. Educ. Nurs. [Internet] 2016 [cited 2019 Nov 18]; 47(6):255-63. DOI: https://doi.org/10.3928/00220124-20160518-06 
23. Krull W, Gusenius TM, Germain D, Schnepper L. Staff perception of interprofessional simulation for verbal de-escalation and restraint application to mitigate violent patient behaviors in the emergency department. J Emerg. Nurs. [Internet] 2019 [cited 2019 Nov 18]; 45(1):24-30. DOI: https://doi.org/10.1016/j.jen.2018.07.001

24. Nickerson M, Morrison B, Pollard M. Simulation in nursing staff development. J. Nurses Staff Dev. [Internet] 2011 [cited 2019 Nov 18]; 27(2):81-9. DOI: https://dx.doi.org/10.1097/NND.0b013e3181a68abd

25. Martins JCA, Mazzo A, Baptista RCN, Coutinho VRD, Godoy S, Mendes IAC, et al. The simulated clinical experience in nursing education: a historical review. Acta Paul. Enferm. [Internet] 2012 [cited 2019 Nov 18]; 25(4):619-25. DOI: https://dx.doi.org/10.1590/S0103-21002012000400022

26. Kim JY, Kim EJ. Effects of simulation on nursing students' knowledge, clinical reasoning, and self-confidence: a quasiexperimental study. Korean J Adult Nurs [Internet]. 2015 [cited 2019 Nov 18]; 27(5):604. DOI: http://dx.doi.org/10.7475/kjan.2015.27.5.604

27. Badir A, Zeybekoğlu Z, Karacay P, Göktepe N, Topcu S, Yalcin B, et al. Using high-fidelity simulation as a learning strategy in an undergraduate intensive care course. Nurse Educ. [Internet] 2015 [cited 2019 Nov 18]; 40(2):E1-6. DOI: https://dx.doi.org/10.1097/NNE.0000000000000134

28. Brasil GC, Ribeiro LM, Mazzo A, Almeida RGS, Martins JCA, Fonseca LMM, et al. Use of the design and self-confidence scales in the assessment of maternal-child realistic simulation. Rev. Enferm. Ref. [Internet] 2018 [cited 2019 Nov 18]; IV Série(19):11726. DOI: https://doi.org/10.12707/RIV18025

29. Buring SM, Bhushan A, Broeseker A, Conway S, Duncan-Hewitt W, Hansen L, et al. Interprofessional education: definitions, student competencies, and guidelines for implementation. Am. J. Pharm. Educ. [Internet] 2009 [cited 2019 Nov 18]; $73(4): 59$. DOI: https://www.ncbi.nlm.nih.gov/pubmed/19657492 